\title{
RESEARCH ON A TRACKSIDE CONCRETE REINFORCEMENT SCHEME OF A SMALL RADIUS CURVE AT A JUNCTION SECTION OF A MODERN TRAM
}

\author{
Zhiqiang WANG ${ }^{1,2 *}$, Zhenyu $\mathrm{LEI}^{1,2}$
}

\begin{abstract}
In order to ensure the normal use of a junction section of a modern tram, this paper mainly studied a trackside concrete reinforcement scheme. Firstly, the entire non-reinforcement system model with a small radius curve composed of rail, fastener, fastener cover, flexible material, asphalt layer and track slab was established using the ABAQUS finite element software, and the stress distribution and deformation state of the asphalt layers of the non-reinforcement system model under the social vehicle load were analyzed. Then, the whole system model of the concrete reinforcement scheme was founded, and the stress and deformation of the asphalt layers under the same load were investigated. Finally, the calculation results of the concrete reinforcement model were com-pared with those of the non-reinforcement model, and the reinforcement effect was studied. The results show that the concrete reinforcement scheme significantly reduces the stress and deformation of the asphalt layers and improves the stress distribution and deformation state of the asphalt layers.
\end{abstract}

Address

1* Institute of Rail Transit, Tongji University, Shanghai, China

2 Shanghai Key Laboratory of Rail Infrastructure Durability and System Safety, Shanghai, China

* Corresponding author: 1733359@tongji.edu.cn

Key words

- Modern tram,

- Junction section,

- Small radius curve,

- Whole system model,

- Concrete reinforcement.

\section{INTRODUCTION}

In recent years, the rail transit of modern tram system has developed rapidly around the world. Compared with the traditional tram, the modern tram has characteristics of high vehicle performance, large passenger capacity, and safety and comfort. It is fast and convenient, and is energy saving with noise reduction and flexible marshalling. The transportation capacity of a modern tram is between a conventional bus and light rail; it can be used as the encryption lines of existing rail transit in large cities, the extension and connection lines of subways, light rail and other rail transit lines in the suburbs, and the public transport backbone lines of satellite cities (new towns) around large cities and small/ medium-sized cities, and is an important part of a urban public transport system (Wu, 2013; Xue et al., 2008).

At the present stage, a large number of scholars have undertaken in-depth research on the track structure of the modern tram. Solkowski et al. (2021) presented tests of selected mecha- nical properties of the cellular polyurethane mats applied on tram tracks. The results showed the remarkable effect of high-cycle fatigue loading on the values of the mechanical parameters. Zhang et al. (2021) investigated the vibration transmission characteristics of different tracks of the modern tram. The results indicated that the rail vibration attenuation rate of embedded track showed an obvious frequency correlation. Di et al. (2021) developed a new embedded track structure for trams with beams erected between two standard prefabricated rail grooves, and verified the applicability and reliability of the new track structure. By establishing the tram dynamics model and the groove rail top profile model, Zhai et al. (2021) studied the operation of safety of a tram system and passenger comfort when the train passed through different negative superelevation curves of the groove rail at different speeds. Liu et al. (2021) carried out research and design of the new fastener system for a modern tram. The theoretical research and production test showed that the fastener system was safe and reliable, and all the indicators met the specification requirements 
that were suitable for the modern tram track system. Yang et al. (2021) analyzed the wear of the wheel tread and rail profile under various track irregularity conditions and different curve radius conditions. The results revealed that a resilient wheel had a better wear reduction effect under the conditions of a poor line. Based on the semi-analytical finite element method, Sun et al. (2020) established an analytical model commonly used in the ballastless track of modern tram and obtained the dispersion curves of ultrasonic guided waves in both free rail and supported rail. By constructing a vertical coupling dynamics model of the modern ballastless track tram, Sun and Feng (2020) studied the specific values of the design and common wheel loads of the corresponding ballastless track. Shan et al. (2020) analyzed the law of the stress history of the subgrade soil of the modern tram's pile-plank structure on the transverse differential settlement of the subgrade through a centrifugal model test. The test results showed that the soil stress history had a significant effect on the differential settlement. Bi et al. (2019) analyzed the static and dynamic behaviors of embedded ballastless tracks by using ANSYS. On the basis of the optimization of the topology and given the requirements of cost, safety, noise, and vibration for the urban rail transit, the structure of the embedded rail trough was optimized.

Modern trams mostly use ground lines, and level crossing sections with municipal roads are frequent. Therefore, the level crossing is the heart of tram transportation. The impact of road vehicles is large, especially for the rail-pavement junction of a small radius curve track, and in many projects worldwide cracking has appeared to different degrees, which has become a common phenomenon. Thus, this paper focuses on a trackside concrete reinforcement scheme in the junction section of a modern tram with a small radius curve. Firstly, the entire non-reinforcement system model with a small radius curve composed of rail, fastener, fastener cover, flexible material, asphalt layer and track slab is established by using the ABAQUS finite element software, and the stress distribution and deformation state of asphalt layers under the social vehicle load are analyzed. Then, the whole model system of the concrete reinforcement is built, and the stress and deformation of the asphalt layers under the same load are studied. Finally, the results of the two models are compared, and the reinforcement effect of the trackside concrete scheme is investigated.

\section{ESTABLISHMENT OF THE SYSTEM MODEL WITH A SMALL RADIUS CURVE}

By using the ABAQUS finite element software, the small radius curve system model is established (Abaqus, 2014). The curve radius of the model is $30 \mathrm{~m}$; the length of the track center line is $6.25 \mathrm{~m}$, and the width of the track section is $4.2 \mathrm{~m}$. The main assumptions of the constitutive model of each component are as follows: the upper layer asphalt and the lower layer asphalt adopt an elastic-plastic constitutive model, and the plastic constitutive model adopts the Drucker-Prager model, which modifies the Mohr-Coulomb yield function and eliminates singular points caused by sharp corners (an opposed to the Mohr-Coulomb model, the corner of the Drucker-Prager yield surface is smooth and conical in the principal stress space) (Zhang, 2009); the other parts adopt an elastic constitutive model. The material parameters of each component are referred to in the literature (Xue et al., 2008; Zhang, 2009; Li, 2019; Zhang et al., 2011) in line with the actual and common situations, as shown in Tables 1-2.

As for the asphalt materials, the "AC" refers to the continuous grading; the " 13 "/ 20 " indicates that the nominal maximum particle size of the mixture is $13 / 20 \mathrm{~mm}$, and the last " $\mathrm{C}$ " is a classification of continuous grading.

The section diagram of the small radius curve system model and the assembly diagram of the overall model are shown in Figures 1-2. As for the model, the fastener part is simulated by spring-damping elements, and the fastener spacing is $0.625 \mathrm{~m}$. The vertical stiffness of the fastener is $40.73 \mathrm{MN} / \mathrm{m}$; the transverse and longitudinal stiffnesses are $8.79 \mathrm{MN} / \mathrm{m}$; the vertical damping is $6361.29 \mathrm{Ns} / \mathrm{m}$; and the transverse and longitudinal damping is $1927.96 \mathrm{Ns} / \mathrm{m}$ (Lei et al., 2019). Tie constraints are used between the surfaces of the other parts (such as asphalt-asphalt, asphalt-concrete, etc.) to better reflect the overall characteristics of the model.

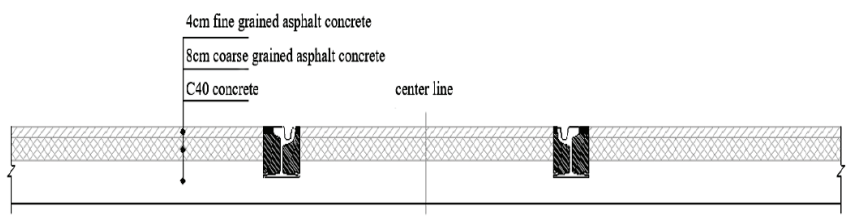

Fig. 1 Section diagram of the system model

Tab. 1 Material parameters of asphalt layers

\begin{tabular}{cccccc}
\hline component & material & elastic modulus $(\mathrm{MPa})$ & Poisson's ratio & yield strength $(\mathrm{MPa})$ & friction angle $\left({ }^{\circ}\right)$ \\
\hline $\begin{array}{c}\text { upper } \\
\text { layer }\end{array}$ & AC-13C & 1400 & 0.35 & 1.1106 & 43 \\
$\begin{array}{c}\text { lower } \\
\text { layer }\end{array}$ & AC-20C & 1200 & 0.3 & 0.9696 & 43.12 \\
\hline
\end{tabular}

Tab. 2 Material parameters of each component, excluding asphalt layers

\begin{tabular}{ccccc}
\hline component & material & elastic modulus $(\mathrm{MPa})$ & Poisson's ratio & $\mathrm{density}\left(\mathrm{g} / \mathrm{cm}^{3}\right)$ \\
\hline groove rail & high carbon low alloy steel & 206000 & 0.3 & 7.85 \\
guard block & natural rubber/synthetic rubber & 7.9 & 0.5 & 1.0 \\
fastener cover & nylon & 8300 & 0.28 & 1.2 \\
slab & C40 concrete & 32500 & 0.2 & 2.36 \\
sealant & polyethylene & 1.15 & 0.35 & 1.15 \\
\hline
\end{tabular}




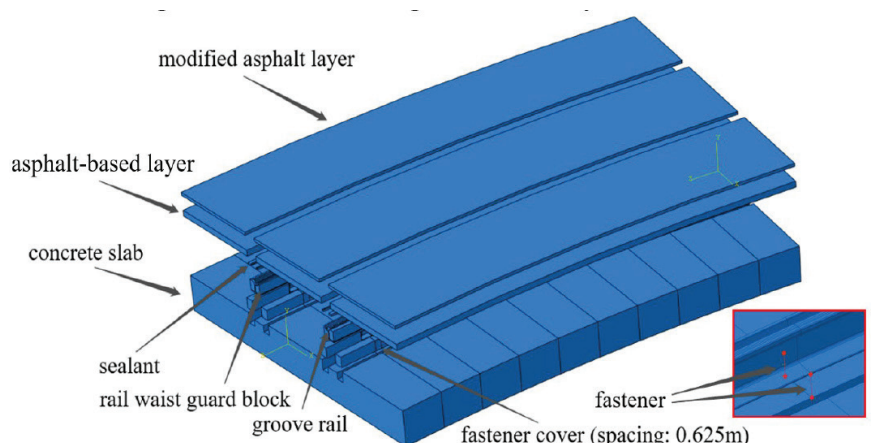

Fig. 2 Assembly diagram of the whole model

For the boundary conditions of the system model, the longitudinal ends of the track are symmetrically constrained; the left and right sides and the bottom surface are fixed constraints. The schematic diagram of the model boundary conditions is shown in Figure 3.

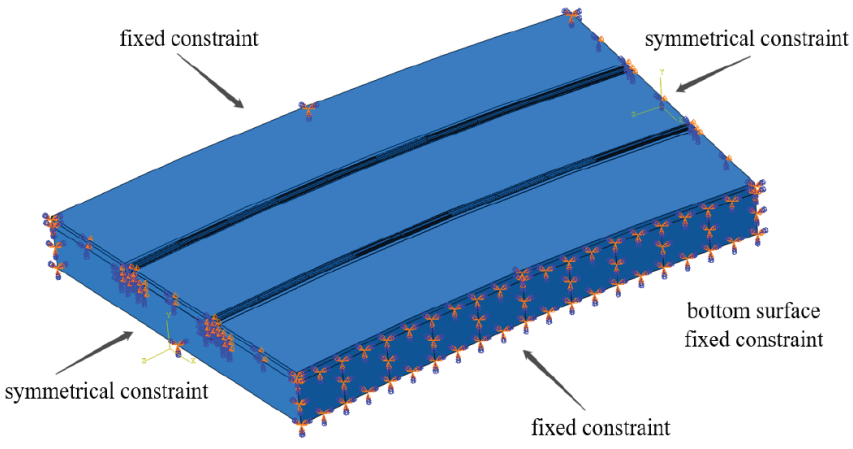

Fig 3 Diagram of the model's boundary conditions

\section{RESEARCH ON THE SMALL RADIUS CURVE SYSTEM MODEL UNDER A SOCIAL VEHICLE LOAD}

\subsection{Setting of the calculation condition}

Referring to the "General specifications for the design of highway bridges and culverts" (JTG D60-2018, 2018), the layout diagram of the social vehicle load of the small radius curve system model selected in this paper is simplified as shown in Figure 4. In this case, the vertical load concentration is $0.40 \mathrm{~N} / \mathrm{mm}^{2}$; the transverse load concentration is $0.24 \mathrm{~N} / \mathrm{mm}^{2}$; and the transverse load points to the rail side and the radius of the load action circle is $0.24 \mathrm{~m}$.

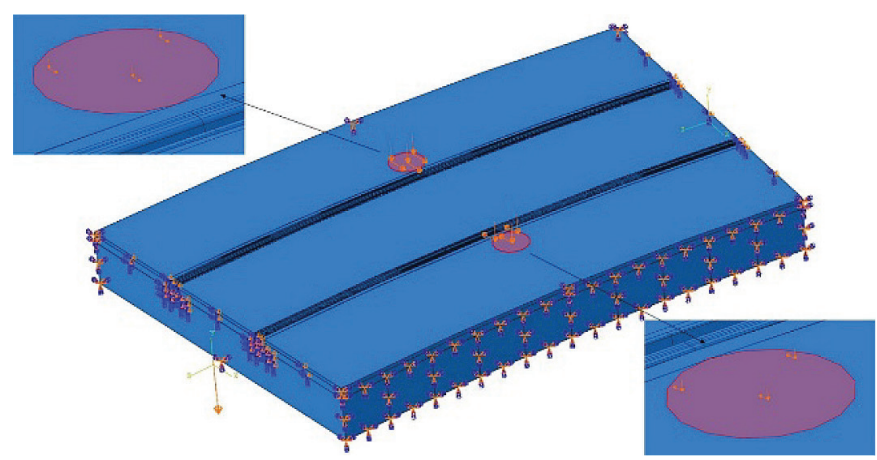

Fig. 4 Load layout diagram

\subsection{Analysis of the calculation results}

In this section, the Mises yield criterion is used to evaluate the strength and stability of the asphalt layers. Firstly, the Mises stress of asphalt layers in the small radius curve system model is analyzed, and the corresponding stress nephograms are shown in Figure 5.

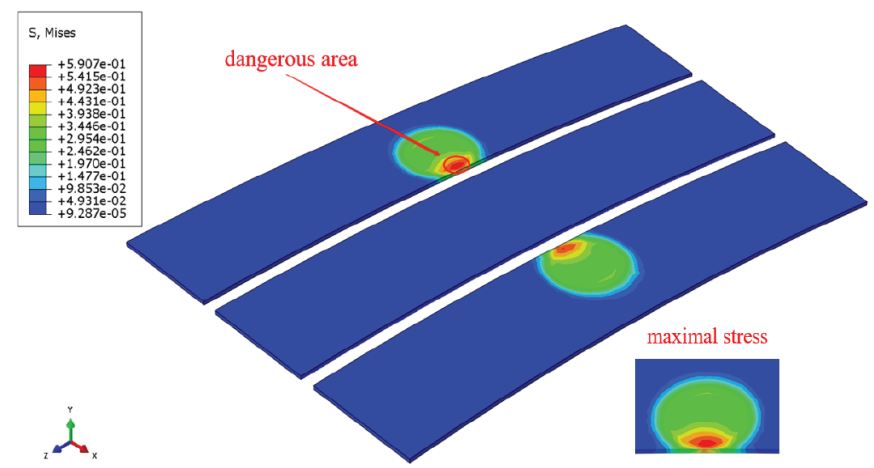

(a) $A C-13 C$ asphalt layer

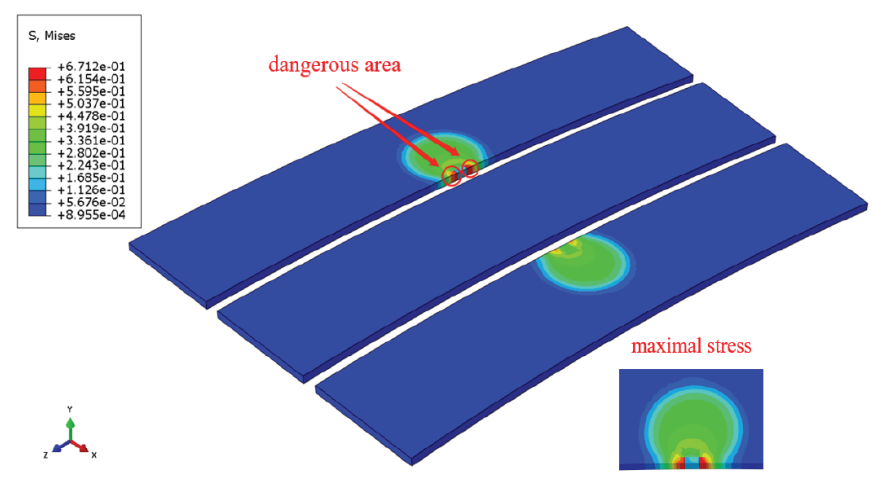

(b) AC-20C asphalt layer

Fig .5 Stress nephograms (unit: $M P a$ )

It can be seen in Figure 5 that the Mises stress nephogram shapes of the asphalt layers (including the AC-13C modified asphalt layer and the AC-20C-based asphalt layer) on the inner rail side and outer rail side are roughly the same and that the Mises stress amplitude of the outer rail side asphalt layer is slightly greater than that of the inner rail side asphalt layer at the same load loading position. Meanwhile, it can be seen from Figure 5 that the maximum Mises stress of the AC-13C modified asphalt layer $(0.591 \mathrm{MPa})$ is located directly above the fastener cover on the outer rail side, which is an approximately elliptical distribution (similar to the literature (Cheng et al., 2019; Meng, 2019)). The reason for the above phenomenon is due to the low rigidity of the fastener cover and the extrusion effect on the edge of the asphalt layer under the horizontal force of the pavement. This location is the most dangerous area of the $\mathrm{AC}-13 \mathrm{C}$ asphalt layer. The maximum Mises stress of the AC-20C-based asphalt layer (0.671 $\mathrm{MPa}$ ) is located at the edge of the asphalt layer on the outer rail side, which is distributed in two strips. The reason is mainly due to the uneven stiffness between the fastener cover and the concrete. This location is the most dangerous area of AC-20C asphalt layer.

By comparing Figures 5 (a) and (b), it can be seen that the maximum Mises stress of the AC-20C asphalt layer is greater than that of the $\mathrm{AC}-13 \mathrm{C}$ asphalt layer, but the maximum Mises stress of the AC-13C and AC-20C asphalt layers does not exceed their corresponding yield strength, which indicates that the asphalt layers 


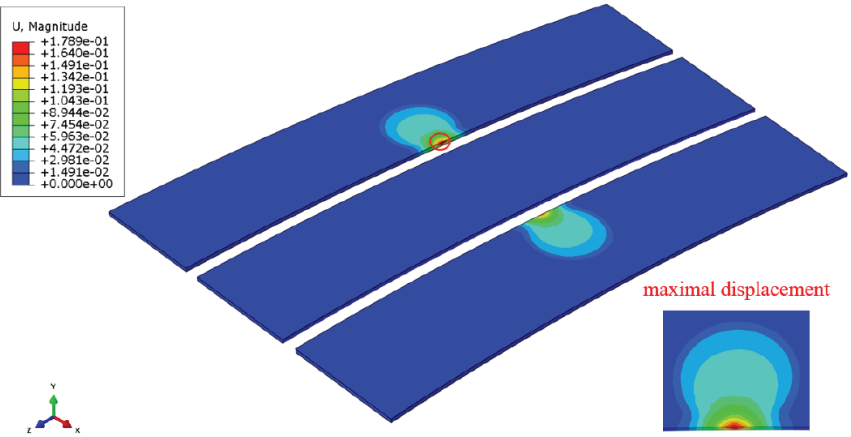

(a) AC-13C asphalt layer

Fig. 6 Displacement nephograms (unit: $\mathrm{mm}$ )

will not undergo plastic deformation under the above vehicle load.

The displacement nephograms of the asphalt layers are shown in Figure 6. It can be seen that the displacement nephogram shapes of the asphalt layers on the inner rail side and the outer rail side are approximately the same; moreover, at the same loading position, the displacement amplitude of the asphalt layer on the outer rail side is slightly larger than that on the inner rail side. Meanwhile, the maximum displacement of the AC-13C asphalt layer, which is located at the edge of the asphalt layer, is $0.179 \mathrm{~mm}$; the maximum displacement of the $\mathrm{AC}-20 \mathrm{C}$ asphalt layer, which is also located at the edge of the asphalt layer, is $0.120 \mathrm{~mm}$; it corresponds to the top of the fastener cover. The reason for the above phenomenon is that the rigidity of the top surface of the fastener cover is smaller than that of the surrounding concrete and that the edge of the asphalt layer is squeezed.

By comparing Figures 6 (a) and (b), it can be seen that the maximum displacement of the $\mathrm{AC}-13 \mathrm{C}$ asphalt layer is greater than that of the AC-20C asphalt layer. Therefore, the deformation of the AC$13 \mathrm{C}$ asphalt layer is more unfavorable under the above vehicle load.

\section{RESEARCH ON THE CONCRETE REINFORCEMENT SCHEME UNDER SMALL RADIUS CURVE CONDITIONS}

\subsection{Establishment of the model}

In a certain range outside the flexible packing material of the system model, the asphalt layers are changed to $\mathrm{C} 40$ concrete, and the connection is established by tie constraints so as to construct the small radius curve system model with a concrete reinforcement, as shown in Figure 7. The boundary conditions of the small radius curve system model with a concrete reinforcement are the same as those of the non-reinforcement system model.

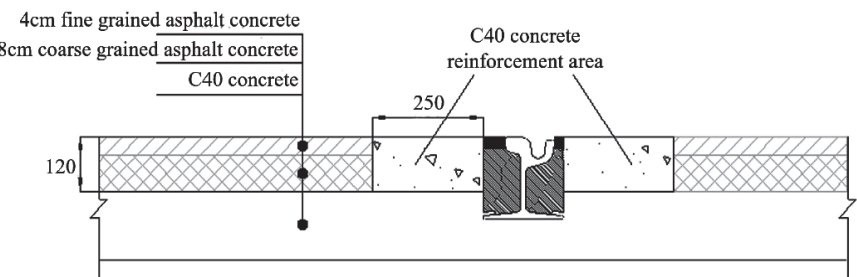

(a) Section diagram of system model

Fig. 7 System model with concrete reinforcement

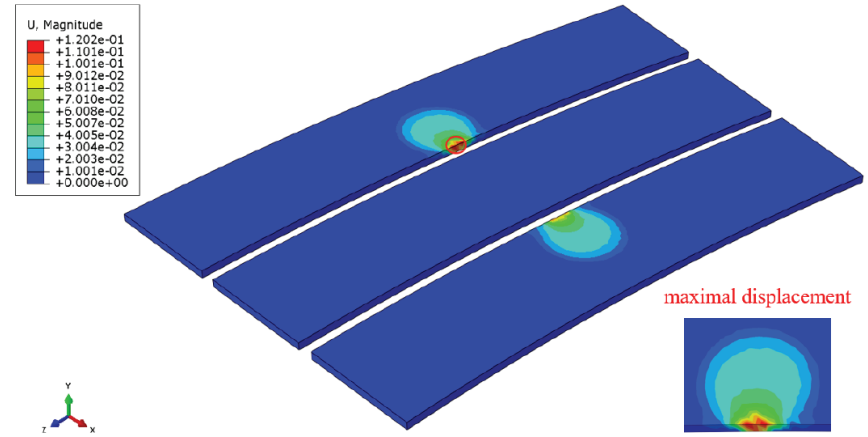

(b) AC-20C asphalt layer

\subsection{Strength and stability analysis}

Firstly, the stress of the asphalt layers is analyzed, and the corresponding Mises stress nephograms are shown in Figure 8.

It can be seen in Figure 8 that the Mises stress nephogram shapes of the inner and outer rail side asphalt layers (including the AC-13C modified asphalt layer and the AC-20C-based asphalt layer) are basically the same and that the Mises stress amplitudes are also roughly the same. Meantime, it can be seen in Figure 8 that the maximum Mises stress of the $\mathrm{AC}-13 \mathrm{C}$ asphalt layer, which is located in the loading area, is $0.370 \mathrm{MPa}$. Compared with the maximum Mises stress of $0.591 \mathrm{MPa}$ of the AC$13 \mathrm{C}$ asphalt layer without any reinforcement (see Figure 5), the maximum Mises stress of the $\mathrm{AC}-13 \mathrm{C}$ asphalt layer decreases by $37.4 \%$. The maximum Mises stress of the AC-20C asphalt layer, which is also located in the loading area, is $0.278 \mathrm{MPa}$. The maximum Mises stress of the AC-20C asphalt layer decreases by about $58.6 \%$ compared with the maximum Mises stress of $0.671 \mathrm{MPa}$ of the AC-20C asphalt layer without any reinforcement (see Figure 5). The main reason is that there is no fastener cover under the asphalt layer due to the existence of the concrete reinforcement layer; it thus avoids the uneven stiffness problem under the asphalt layer, so the peak stress of the asphalt layer is greatly reduced.

By comparing Figures 8 (a) and (b), it can be seen that the maximum Mises stress of the $\mathrm{AC}-13 \mathrm{C}$ asphalt layer is greater than that of the AC-20C asphalt layer, but the maximum Mises stress of the AC-13C and AC-20C asphalt layers does not exceed their corresponding yield strength; this indicates that the asphalt layers will not undergo plastic deformation under the above vehicle load.

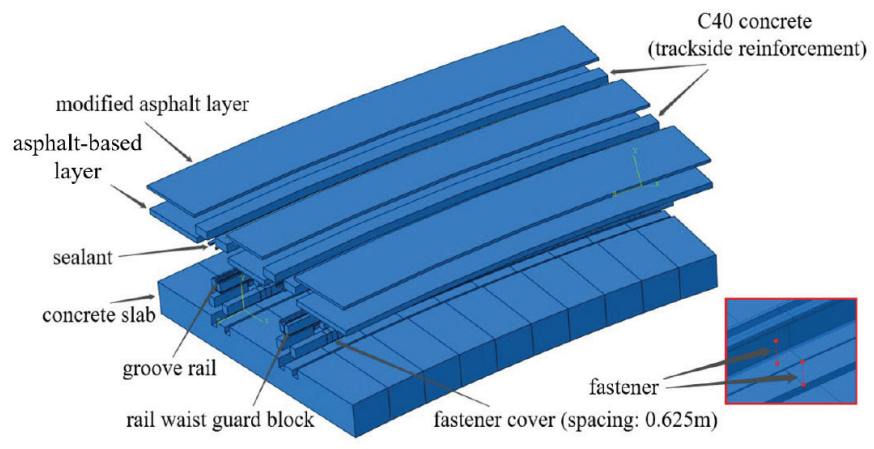

(b) Assembly diagram of the whole model 


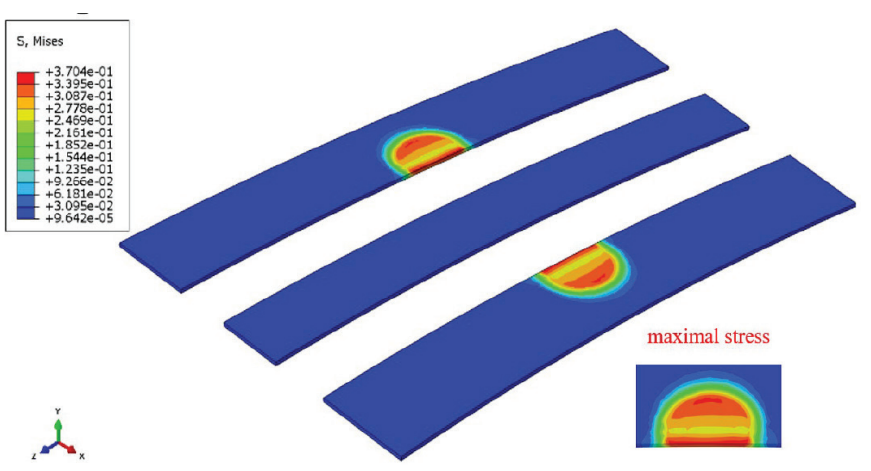

(a) AC-13C asphalt layer

Figure 8. Stress nephograms (unit: $M P a$ )

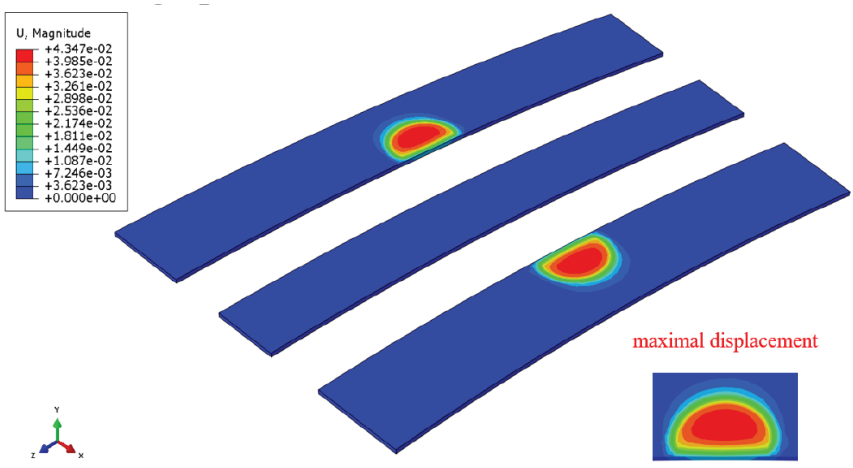

(a) AC-13C asphalt layer

Fig. 9 Displacement nephograms (unit: $\mathrm{mm}$ )

The displacement nephograms of the asphalt layers are shown in Figure 9. It can be seen that the displacement nephogram shapes of the asphalt layers at the inner rail side and the outer rail side are roughly the same and that the displacement amplitudes are almost the same. Compared with the maximum displacement of $0.179 \mathrm{~mm}$ of the $\mathrm{AC}-13 \mathrm{C}$ asphalt layer under the non-reinforcement scheme, the maximum displacement of the $\mathrm{AC}-13 \mathrm{C}$ asphalt layer under the concrete reinforcement scheme is only $0.043 \mathrm{~mm}$, which is reduced by $76.0 \%$. Compared with the maximum displacement of $0.120 \mathrm{~mm}$ of the $\mathrm{AC}-20 \mathrm{C}$ asphalt layer under the non-reinforcement scheme, the maximum displacement of the AC-20C asphalt layer under the concrete reinforcement scheme is only $0.031 \mathrm{~mm}$, which is reduced by $74.2 \%$. The above phenomenon is due to the stiffness uniformity under the asphalt layer and the constraint of the concrete reinforcement layer on the asphalt transverse deformation.

By comparing Figures 9 (a) and (b), it can be seen that the maximum displacement values of the AC-13C and AC-20C asphalt layers are small; therefore, the deformation of the asphalt layers is within the safe range under the above vehicle load.

\section{CONCLUSIONS}

(1) As for the concrete reinforcement scheme, the maximum Mises stress of the AC-13C asphalt layer decreased by $37.4 \%$ compared with that of the AC-13C asphalt layer without any reinforcement; the maximum Mises stress of the AC-20C asphalt layer decreased by $58.6 \%$ compared with that of the AC-20C asphalt layer without any reinforcement. The main reason is that there is no fastener cover under the asphalt layer due to the existence of the

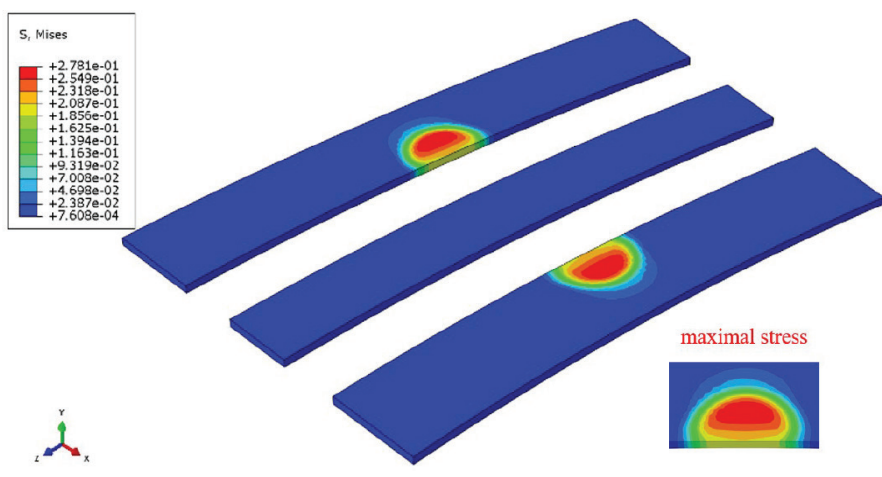

(b) AC-20C asphalt layer

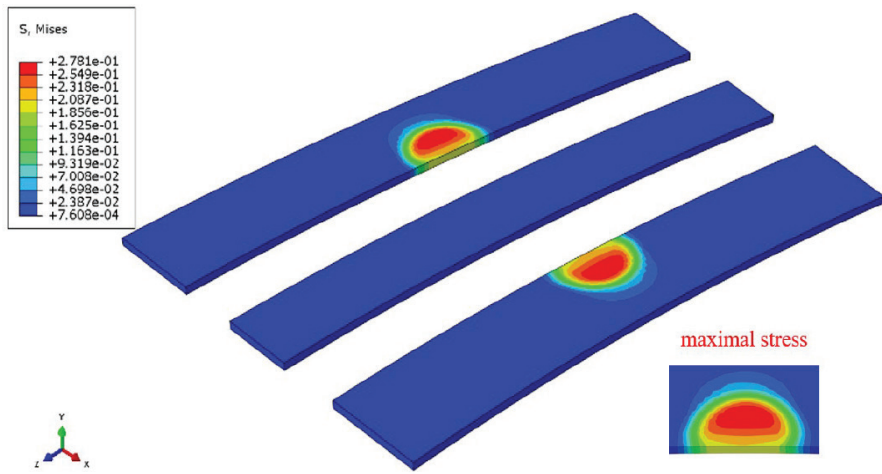

(b) AC-20C asphalt layer

concrete reinforcement layer; it thus avoids the uneven stiffness problem under the asphalt layer, so the peak stress of the asphalt layer is greatly reduced.

(2) Compared with the maximum displacement of the AC$13 \mathrm{C}$ asphalt layer of the non-reinforcement system model, the maximum displacement of the AC-13C asphalt layer under the concrete reinforcement is reduced by $76.0 \%$. Compared with the maximum displacement of the AC$20 \mathrm{C}$ asphalt layer of the non-reinforcement system model, the maximum displacement of the AC-20C asphalt layer under the concrete reinforcement is reduced by $74.2 \%$. The above phenomenon is due to the stiffness uniformity under the asphalt layer and the constraint of the concrete reinforcement layer on the asphalt transverse deformation.

(3) Considering both the stress and displacement, it can be concluded that the concrete reinforcement scheme has significant advantages over the non-reinforcement scheme. Therefore, in order to avoid problems such as the asphalt layer cracking and to prolong the service life of a track structure, the concrete reinforcement scheme is more suitable for small radius curve lines and sections with a large traffic volume.

\section{Acknowledgements}

This study was supported by the National Natural Science Foundation of China (11772230). 


\section{REFERENCES}

Abaqus. 2014. 6.14 documentation. Dassault Systemes Simulia Corporation, Providence, USA.

Bi, L. X. - Liu, W. X. - Xing, M. T. - Zhao, P. R. - Liu, X. Y. 2019. Optimal design for internal structure of embedded rail trough. Journal of Southwest Jiaotong University 54(6): 12271234.

Cheng, Y. - Xu, Z. L. - Zhu, J. Y. - Zhang, W. Q. - Meng, D. 2019. Research on rail waist block structure of modern tram. Urban Mass Transit 22(S1): 76-81.

Di, Y.F. - Dong, C. Z. - Yin, H.T. - Wang, W.D. - Zeng, Z. P. - Huang, X. D.- Wang, D. 2021. Research on optimal design of a novel frame embedded track structure for tram. Bulletin of Science and Technology 37(9): 103-108.

JTG D60 - 2018. 2018. General specifications for design of highway bridges and culverts. China Communications Press, Beijing, China.

Lei, Z. Y. - Wang, Z. Q. - Li, L., Geng - C. Z. 2019. Rail corrugation characteristics of the common fastener track in metro. Journal of Tongji University (Natural Science) 47(9): 13341340.

Li, J. P. 2019. Dynamic performance of embedded track structure of modern tram. Shijiazhuang Tiedao University, Shjiazhuang, China.

Liu, X. F. - Li, J. X. - Ma, J. J. 2021. Research on new type of fastener system on modern tram rail. Modern Urban Transit, (4): $42-48$.

Meng, D. 2019. Design optimization of the bearing capacity for tram fastener cover. Urban Mass Transit 22(S1): 67-72.

Solkowski, J. - Gorszczyk, J. - Malicki, K. - Kudla, D. 2021. The effect of fatigue test on the mechanical properties of the cellular polyurethane mats used in tram and railway tracks. Materials 14(15): 4118.
Shan, Y. - Lu, Y. - Zhou, S. H. - Wang, B. L. 2020. Centrifugal model test study on lateral differential settlement between the tram pile-plank subgrade and the road subgrade. Chinese Journal of Rock Mechanics and Engineering 39(5): 10491060 .

Sun, K. - Chen, H. P. - Feng, Q. S. - Lei, X. Y. 2020. Propagation characteristics of ultrasonic guided waves in tram rails. Structural Engineering and Mechanics 75(4): 435-444.

Sun, K, Feng, Q. S. 2020. Research on the train load value of ballastless track of modern tram. Journal of Railway Science and Engineering 17(5): 1113-1120.

Wu, Q. G. 2013. Research on the emphasis and difficulties of modern tramcar system development and its countermeasures. Journal of Railway Engineering Society 30(12): 89-92+98.

Xue, M. G. - Yang, L. F. - Cheng, J. 2008. Modern trams: characteristics and development both at home and abroad. Urban Transportation of China 6(6): 88-91+96.

Yang, Y. - Shen, J. - Wang, K. M. - Zhan, J. 2021. Research on low floor tram wheel and rail wear under resilient wheels. China Mechanical Engineering 32(4): 439-445.

Zhai, B.B. - Ren, Z. S - Qu, C. 2021. Dynamic performance of tram passing through groove rail with negative superelevation curve. Urban Mass Transit 24(7): 129-134.

Zhang, L.J. 2009. Study on constitutive relation and rutting prediction method of asphalt mixture based on creep test. South China University of Technology, Guangzhou, China.

Zhang, L. J. - Zhang, X. N. - Chen, Y. K. 2011. Visco-elastoplastic constitutive model of deformation for asphalt mixture. Journal of Wuhan University of Technology (Transportation Science and Engineering) 35(2): 289-292.

Zhang, X. - Li, X. - Hao, C. X. - You, Y. C. - Chen, W. J. - Pu, Z. - Xu, Y. J. 2021. Experimentation on vibration transmission characteristics of modern tram tracks. Journal of Southwest Jiaotong University 56 (1): 75-83. 